\title{
A Computer-Accessed Microfiche Library
}

\author{
R. G. J. ZIMMERMANN: Department of Engineering-Economic Sys- \\ tems, Stanford University, Stanford, California. At the time this article \\ was written, the author was a member of the Technical Staff, Space \\ Photography Laboratory, California Institute of Technology, Pasadena, \\ California.
}

This paper describes a user-interactive system for the selection and display of pictorial information stored on microfiche cards in a computercontrolled viewer. The system is designed to provide rapid access to photographic and graphical data. It is intended to provide a library of photographs of planetary bodies and is currently being used to store selected. Martian and lunar photography.

\section{INTRODUCTION}

Information is often most usefully stored in pictorial form. Photography, for example, has become an important means of recording data, especially in the sciences. A major reason for this importance is that photographs can be used to record information collected by instruments and not normally observable by the unaided eye. Such photographs, especially in large quantities, may present a barrier to their use because of the inconvenience of reproducing and handling them. It is apparent that a system to compactly store and to speed access to these photographs would be very useful. Such a system, utilizing a microfiche viewer directly controlled by a user-interactive computer program, has been developed to support a library of photographs taken from space.

In the past fifteen years, the National Aeronautics and Space Administration has conducted many missions to photograph planetary bodies. These missions have provided millions of pictures of the earth, moon, and Mars. A large number of additional pictures are expected to be taken in the near future. The Space Photography Laboratory of the California Institute of Technology is establishing, under NASA auspices, a microfiche library of a selection of these photographs. The library currently contains the photographs of Mars taken by the Mariner 9 spacecraft as well as lunar photographs taken by the Lunar Orbiter series. The library is expected to be expanded as time and resources permit. It has been operating, with various versions of the control program, since June 1972. The program is currently being further developed by Mr. David Neff and Miss Laura Hor- 
ner of the Space Photography Laboratory at the California Institute of Technology.

\section{HARDWARE}

The photographs are kept on 105-by-148mm microfiche cards, sixty frames to a card. This format provides the least reduction of any standard microfiche format and was used to retain the highest possible resolution. The cards are displayed by a microfiche viewer (Image Systems, Culver City, California) which can store up to about 700 cards and has the capability of selecting a card and displaying any frame on it within a maximum of about four seconds. (Throughout this paper, "viewer" will be used to refer to the microfiche viewing device.) The viewer can be equipped with a computer interface which allows the picture display to be directly computer controlled. An installation consists of the viewer with interface, any standard input/output (I/O) terminal, and the control program, running, in this case, on a time-shared computer. The terminal is used for communication with the control program. The user enters all commands by typing on the terminal keyboard. The viewer is designed to be plugged in between the computer and I/O terminal. The computer transmits all information on the circuit to which normally (without the viewer) only the terminal is attached. This information includes the viewer picture display control codes which are recognized and intercepted by the viewer. All other information is passed on to the terminal. No further special equipment is necessary.

The system described has been implemented on a Digital Equipment Corporation System 10 medium-scale computer with a time-sharing operating system. The program is written mainly in FORTRAN with some assembly language subroutines. It runs in $12 \mathrm{~K}$ words ( $36 \mathrm{bits} /$ word) of core memory. The program will not run without conversion on any computer other than the DEC System 10.

\section{SOFTWARE}

The control program is user-interactive, that is, it accepts information and commands from the user. These commands allow him to indicate what he desires and to control the action taken by the program. The program permits the user to indicate what characteristics he wishes the pictures to have, selects the pictures that satisfy his criteria, and then allows him to control the display of the selected pictures and to obtain any additional information he may need to interpret the pictures. To guide the user, instructions for use of the system, as well as other information the user may need, are displayed on the viewer as they are required. All user responses are extensively checked for validity. Any uninterpretable response is rejected with a message indicating the source of the trouble, and may be reentered in corrected form. It is always possible to return to a previous state, so it is impossible to make a "catastrophic" error. In designing the 
system, particular attention was paid to integrating the viewer and computer to utilize the unique capabilities of each. For example, most instructions are presented on the viewer where they can be shown quickly and can be scanned easily by the user. Only short messages need to be sent and received by the I/O terminal.

\section{Data Base}

A picture is described by a number of characteristics, called parameters. For every picture stored in the viewer, the value for each of these parameters is stored in a disc file. In this application, parameters are mainly used to describe characteristics that are available without analyzing the picture for content. In science, these are the experimental conditions-such as viewing and lighting conditions for space photography.

Because space photographs are taken by missions with different objectives and equipment, it was necessary to design a library system to include pictures with widely varying selection characteristics. In order to accommodate sets of pictures with widely differing characteristics, without wasting storage space or requiring the elimination of useful descriptors, the computer storage has been structured to allow pictures to be grouped into picture sets, each of which is described by its own set of parameters. Conversely, any group of pictures for which the same selection parameters are used forms a picture set. The characteristics of each such set of pictures are also stored and the program reconfigures itself to these characteristics whenever a new picture set is encountered. Such an organization allows the control program to be used on groups of totally different kinds of pictures.

\section{Operation}

In selecting a picture set the user is guided along a series of decisions presented on the viewer. At each step the control program directs the viewer to display a frame with a set of possible choices. The user enters his response on the I/O terminal and the control program uses this response to determine which frame the viewer should be commanded to display next. When the user has selected a set, he is shown the available parameters and apppropriate values for these parameters. After he has specified acceptable values for the parameters he is interested in, the computer program compares these values with the known values in its records for the picture set. The pictures selected by the program are then available for display. As will be described, the user may, at any time, select another picture set or change his parameter specifications. He may also indicate which pictures of those selected by the computer during the comparison search he wishes to have remain available after the next comparison search. This allows comparison of pictures in different picture sets. Appendix 1 shows an example of a typical search.

The action of the control program can be separated into five phases of 
operation, each with a distinct function. The functions of three of these phases involve user interaction. Transfer between phases may also be accomplished by user command. A different group of commands is employed for each of the user-interactive phases. In addition, there is a group of commands which may be used any time a user response is requested; they are listed in Appendixes 3 and 4. There are no required commands or sequences of commands. The user proceeds from one phase to another as he desires. In each phase allowing user interaction, the user can enter any valid command at any time. Figure 1 shows the phases and possible transfers between phases. A more detailed description of what occurs in each phase will be given after the data organization is described.

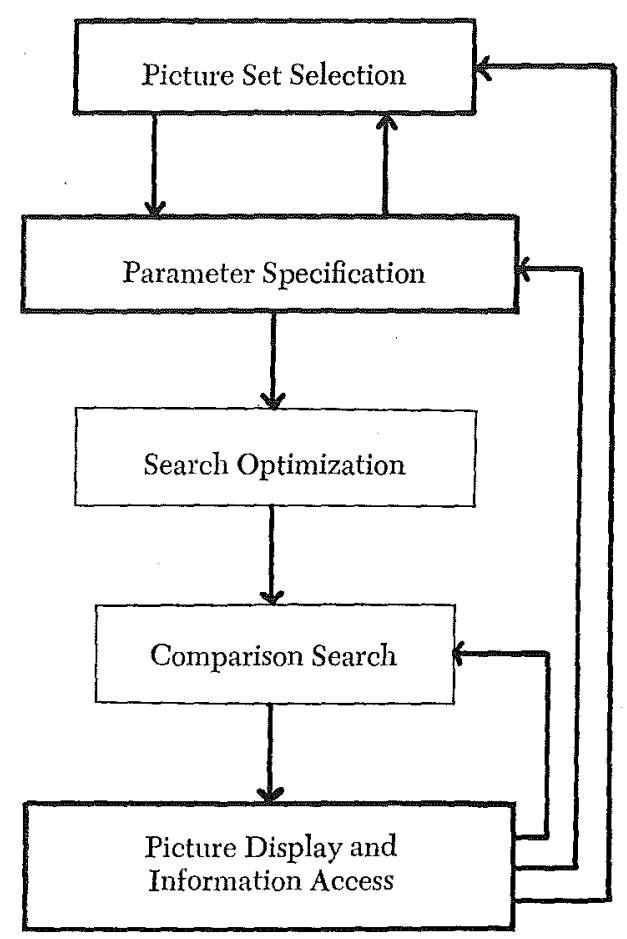

Bold lines enclose user-interactive phases. Arrows indicate possible directions of control transfer; bold arrows are control transfers made by user commands.

Fig. 1. Phases and Control Transfers.

\section{DESCRIPTION OF SOFTWARE}

\section{Data Base Organization}

As has been stated, the pictures of the library are grouped into picture sets. The data base may contain any number of picture sets. Each such set has a picture file associated with it. This picture file is on disc storage and 
contains all the known information stored for a set of pictures. Each picture in the set has an associated picture record in the file. In addition, the first record in a picture file, known as the format record, contains all the file specific information about that file. Whenever a new picture file is called for, the format record for that file is read from disc storage into main memory and kept for reference. Figure 2 shows the organizational structure of the data base.

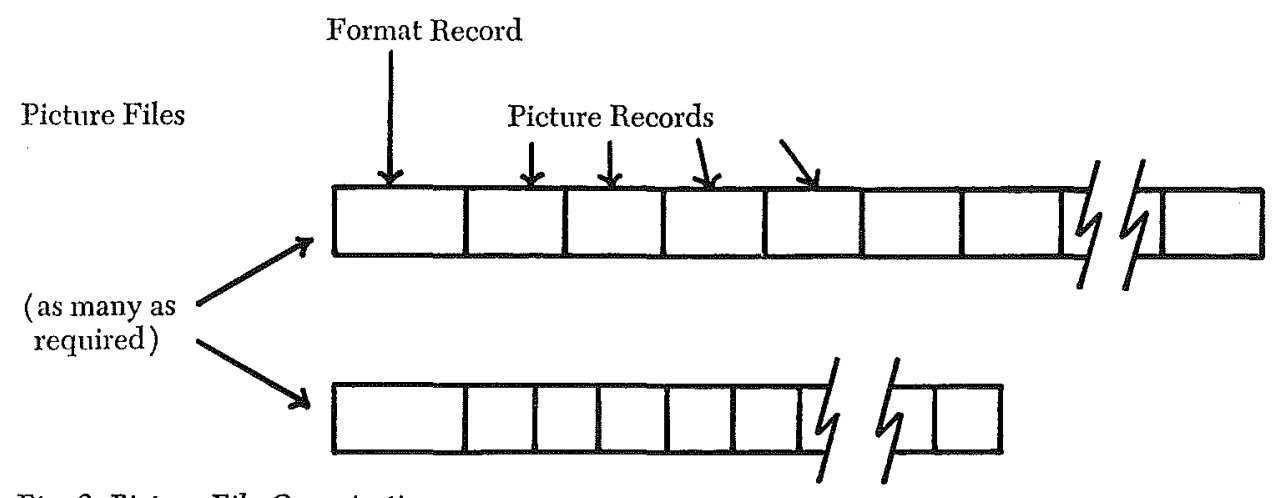

Fig. 2. Picture File Organization.

Picture records consist of a fixed- and a variable-length portion. The variable-length portion contains the known values, for the associated picture, of the specification parameters. Since the number of parameters can vary from file to file, the length of this portion varies from file to file. (However, all picture records within a particular file have the same length and form.) The maximum number of parameters for a system is determined by array dimensions set when the program is compiled. Currently these dimensions are set for a maximum of fifty parameters for any file in the system. The fixed-length portion contains (generally) the same type of information for all files. It includes the information needed to display a picture and to obtain interpretive information. When, during the comparison search, a picture is selected on the basis of information in the variable data, the fixed-length portion is copied into a table and kept for use during the picture display phase. Each selected picture is represented by an entry in this table. The contents of the fixed-length portion are presented in Table 1. As an example, the contents of a picture record for the Mariner 9 photographs are given in Appendix 5.

A picture file's format record describes the file by all characteristics that are allowed to vary from file to file. The format records for all picture files have the same form; each is divided into a number of fields supplying information for a particular function. These fields can be separated into two categories: those which describe the picture records and those which apply to the file as a whole. For fields of the first type, each parameter has an entry in the field. For example, one such field contains the location, in 
Table 1. The Fixed-Length Portion of a Picture Record

Field

Fiche Code

File Name

Picture Number

Unit Number

ID Number

Auxiliary Codes ( 3 fields)
Use

Control code output by the control program to the viewer to display the frame associated with this picture record.

The file name of the picture file; this and the picture number uniquely identify the picture record and allow it, and specifically the contents of the variable portion, to be refound.

A sequence number assigned each picture record in the file in increasing order.

The viewer that the picture associated with this picture record is stored in.

The identification number referred to by the user. If the picture has been given an ID number by which it is commonly known, it will be kept in this field.

Viewer control codes for frames containing different versions of, or auxiliary data for, the picture. The actual contents of these fields vary with the picture file as determined from the contents of the format record of that file.

a picture record, of the value for each of the parameters. Another field has a ten-letter description of each parameter. See Appendix 2 for a description of the format field.

\section{Operation of the Control Program}

The following is a brief technical description of the control program; detailed documentation is available.

The control program is modularly constructed. Each phase consists of a major subroutine and its subsidiary subroutines. At the completion of a phase, control is transferred to a main program which determines which phase is to be performed next and transfers control to it. The user-interactive (interrogation) subroutines ask for a user response, attempt to interpret the response and perform the desired function, then ask for another response.

An important subroutine used by all the interrogation subroutines collects the characters of the user response into groups of similar characters to form alphabetic keywords, numbers, punctuation marks, relational operators, etc. When an interrogation subroutine is ready for a user request, it calls this "scanning" subroutine. The scanning subroutine outputs an asterisk, indicating it is ready, to the user I/O terminal. The scanning subroutine supplies the groups of characters, along with a description of the group, to the interrogation subroutine. The interrogation subroutine then attempts to interpret the character groups by comparing them with acceptable responses. If the response is not in one of the acceptable forms, an error message is given to the user and he can try again. The error message includes an indication of where the error was found and describes the error.

Some commands do not need to be interpreted by the interrogation subroutines; the function they request is the same throughout the program. These are called immediate commands and are listed in Appendix 3. These 
commands are interpreted, and their functions performed, by the scanning subroutine.

\section{Picture Set Selection}

In selecting a picture set the user is asked to make a series of decisions. For each decision, a frame listing the possible choices is displayed on the viewer. All possible decisions form an inverted tree structure (see Figure 3 ). The user may also return to a previous decision point. The tree structure is implemented in a table in computer storage. There is an entry in this table corresponding to each decision point in the tree. When a decision

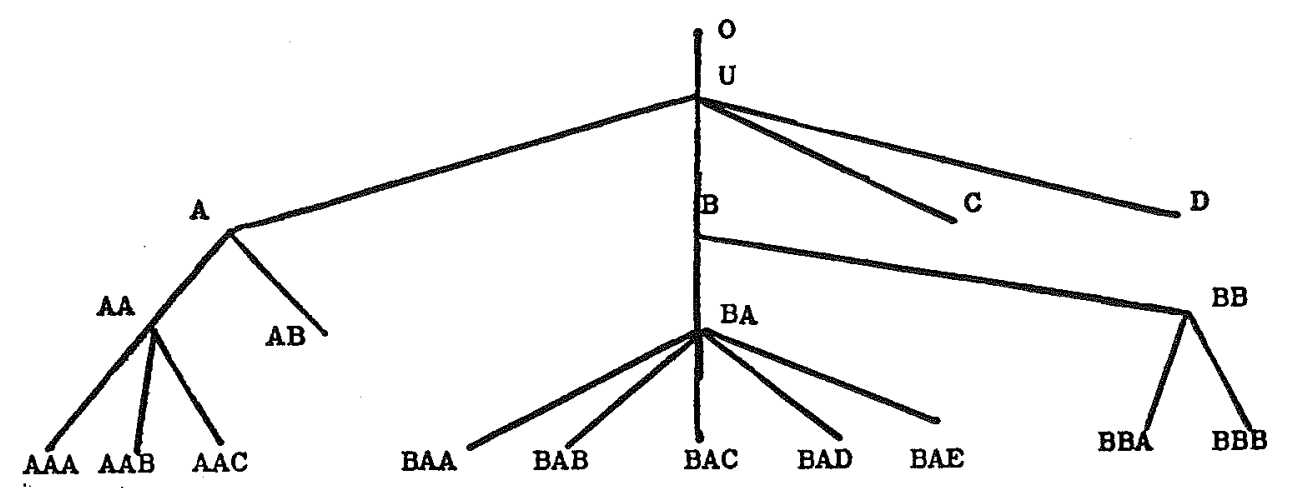

A Martian

AA Orbital - Flyby

AAA Mariner IV

AAB Mariner VI, VII

AAC Mariner IX

$A B$ Surface - Viking

B Lunar

BA Orbital - Approach

BAA Apollo Hand Held

BAB Apollo Metric

BAC Apollo Pan

BAD Lunar Orbiter

BAE Ranger

BB Surface

BBA Apollo

BBB Surveyor

C Venus - F1yby

D Mercury - Flyby

Fig. 3. Example of a Tree. 
is made, the entry corresponding to the new decision point is obtained. An entry at the bottom of the tree identifies the picture file associated with the picture set selected. In general, an entry contains: (1) the viewer control code of the frame displaying the choices; (2) a pointer to the entry from which this node was reached; (3) the number of possible decisions which can be made at this decision point (to check for valid decisions); and (4) pointers to the entries for the decision points reached.

\section{Parameter Specification}

Once the user has made a decision selecting a set of pictures, he is presented with a list of the available parameters and acceptable values for them. For each parameter in which the user is interested, he specifies the parameter number and the values or range of values acceptable to him. This information is stored in two tables which are referred to when the comparison search is made. One table, the parameter table, contains an entry for each parameter specified. This table is cleared whenever a new picture set is called for. An entry in the table includes: (1) the parameter number; (2) a code indicating which of several methods is to be used in processing the parameter; (3) a code providing information on how the user-specified values are to be interpreted; and (4) a pointer to the location in a second table, the values table, where the first of the specified values is stored. All additional values are placed in the values table following the addressed value. The processing code (number (2) above) allows each parameter to be processed by a unique method. A standard method for a given parameter is kept in a field of the format record. The user can also specify a method other than the standard one. If an entry already exists for a just-entered parameter, the old entry is updated rather than a new one created.

\section{Search Optimization}

This phase determines the most efficient way to conduct the comparison search from among a set of alternatives. Whenever possible, the search is restricted to only a part of the picture file. For each picture file there is a number of parameters for which additional information is available. Specifically, if a list of pictures ordered by increasing value of a parameter is available, the pictures which have a particular value of that parameter can be found more quickly through this list than by searching through the whole file for that value of the parameter. If the position, in this ordered list, of the picture at the low end of a range of values (of the parameter it is ordered on) can be found easily, the search can be started at this point and need only be continued until the picture at the high end has been reached. Note that the picture records for the intervening pictures must nonetheless be compared with the user specifications since the restriction is only made on the basis of one parameter whereas more than one may have been specified. 
A binary search is the method used to search the list for the first picture in a range of values. To use this method, of a set of $n$ picture records the $n / 2$ th is chosen and its value of the parameter is compared with the desired one. Since the list of records is in order of the value of this parameter, it is clear in which half of the list a picture with the desired value of the parameter would have to be. This interval can then be divided and the process continued until the remaining interval consists of only one picture. The main picture file is itself usually arranged in order of at least one parameter. For other parameters, control lists of picture numbers ordered by value of these parameters can be used for binary searches. However, it is not practical to create these lists for all parameters as they require a fair amount of storage. An entry in such a list contains two words, the value of the parameter and the picture number of the corresponding picture. Picture number is a sequence number which determines the position of the picture record relative to the beginning of the picture file.

Each picture file has a table in its format record containing identifiers for the parameters for which the binary search technique can be used. If more than one of these has been specified (as stored in the parameter table), it must be determined which parameter restricts the search the most. To do this the upper and lower limits of the specified values of each such parameter are found (from the values table), and from this the expected number of picture records to be compared is computed. This number is multiplied by a factor indicating the speed of the type of search to be used relative to the speed of the simplest type of search. The parameter with the lowest expected elapsed time of search is selected for the search.

\section{Comparison Search}

For each picture to be compared, the appropriate picture record is found and specified parameter values are compared with those in the picture record. A control list, selected in the search optimization phase, may be used to determine which picture records are to be compared. For each selected picture an entry containing a portion of the picture record is made in a picture table. The picture table has a limited capacity which is set when the program is compiled. For our application there is currently room for up to 100 entries. If the picture table is filled before the search is finished, the search is suspended and can be continued by a command in the display phase.

\section{Picture Display, Information Access}

This phase accepts commands to control display of the selected pictures and provide access to interpretive information. The picture table entries provide the information needed, either directly or by referring back to the picture record. Any of the selected pictures can be viewed at any time. In addition, the user can "mark" preferred pictures to differentiate them from the others. These marked pictures are set apart in the sense that 
many viewing and information access commands refer optionally to only these pictures.

The pictures themselves are the primary source of information, but the user will often want information that is not available from the picture in order to interpret the picture. There are commands that request the control program to type out on the $\mathrm{I} / \mathrm{O}$ terminal the information in a picture record. These commands optionally refer to the picture currently displayed, the marked pictures, or all the selected pictures. Other commands call for the display of data frames associated with a picture. These frames can contain large volumes of data that need not be kept in computer storage. The viewer control codes for these frames are kept in the picture table. The keyword commands to display data frames can vary from file to file. The valid commands for a file are kept in the file's format record.

There are other commands to transfer control to other phases and to keep desired pictures available for display with those selected by the next comparison search. There is also a provision for adding file specific commands to perform any other function. The commands and their functions are listed in Appendix 3.

\section{PERFORMANCE AND COSTS}

A typical simple search consisting of logging in, picture selection, parameter specification, search, and display might take five to ten minutes and cost one to two dollars for compute time. Most of this is time spent by the user in entering commands. Command execution is usually almost immediate as it does not involve a major amount of computation. Most of the compute time is accumulated during the comparison search phase. To search through the entire Mariner 9 picture file of around 7,000 pictures (about 200,000 words) takes about forty seconds elapsed time and costs about two dollars. A more typical search, however, will allow some search optimization and cost about thirty cents with an elapsed time of ten seconds. Of course, these figures should only be used as estimates, even for other DEC System 10 systems, as elapsed time depends on system load and this, as well as the rates charged, varies considerably. Total monthly compute costs for a system depend entirely on use. Likewise, storage costs depend on actual storage space used. For the 200,000-word Mariner 9 file our cost is about seventy-five dollars per month. Only the most-used picture files actually need be kept on disc; the rest can be copied from magnetic tape if they are needed. All files are backed up on magnetic tape in any case. The rates listed in this paper are those charged by our campus time-sharing system. DEC System 10 computer time is available from commercial firms at somewhat higher rates.

The cost for a microfiche viewer with computer interface (Image Systems, Culver City, California, Model 201) is around \$7,000. A thirty-characters-per-second I/O terminal sells for $\$ 1,500$ and leases for $\$ 90$ per month. In addition, an installation may require a microfiche camera and 
other photographic equipment and supplies. Photographic services are also available from the viewer manufacturer. The hardware cost for an independent system implemented on a minicomputer with $12 \mathrm{~K}$ to $20 \mathrm{~K}$ of core and five million words of disc memory is estimated at an additional $\$ 30,000$ (exclusive of development and photographic costs).

\section{IMPLEMENTING A LIBRARY SYSTEM}

In implementing a library system to use the hardware and software described in this paper, two major areas of effort are required. First, the pictorial information must be converted to microfiche format; that is, it must be photographed, or possibly rephotographed if already in photographic form. In addition, a computer data base must be created. If information about the photographs is already available in computer-readable form, this involves writing a program to convert the data to the structure required by the control program. If this type of information is not available, the pictures may need to be investigated and the information coded, and presumably punched onto computer cards, for further processing. The major difficulties we encountered were coordinating the photographic and data base generation tasks, achieving the high resolution we required to retain the detail of the original photographs, and in using early versions of the microfiche viewer (which had a tendency to jam cards).

\section{CONCLUSION}

A system for rapid access to pictorial information, the Computer Accessed Microfiche Library (CAML), has been described. CAML has been designed to integrate, in an easy-to-use system, the storage capacity and capability for fast retrieval of a special microfiche viewer with the manipulating ability and speed of a computer. It is believed that this system will help overcome the barriers to the full utilization of photographs in large quantities, as well as have applications in the retrieval of other types of pictorial information.

\section{ACKNOWLEDGMENTS}

The work described in this paper was supported by NASA grant \#NGR 05-002-117. The author is grateful to Dr. Bruce Murray and the staff of the Space Photography Laboratory at Caltech for their support and advice; he also wishes to acknowledge the efforts of Mr. James Fuhrman, who assisted in the programming task and contributed many valuable ideas.

\section{APPENDIX 1}

The following is an example of a typical search. Numbers in the left margin indicate when a new frame is displayed on the viewer. These were added later to clarify the interaction between viewer and terminal. User responses and commands are identified by lines beginning with an asterisk. (The control program types asterisks when it 
is ready for input.) In this demonstration, most keywords were completely typed out. It is possible, however, to abbreviate any keyword to the shortest form that will be unique among the acceptable keywords.

After the user enters a standard "log in" procedure to identify his account number and verify that he is an authorized user of this account, the control program is automatically initiated. The viewer displays a picture (1) of the installation and the user is asked to enter his name. The name, charges, and time of use will later be added

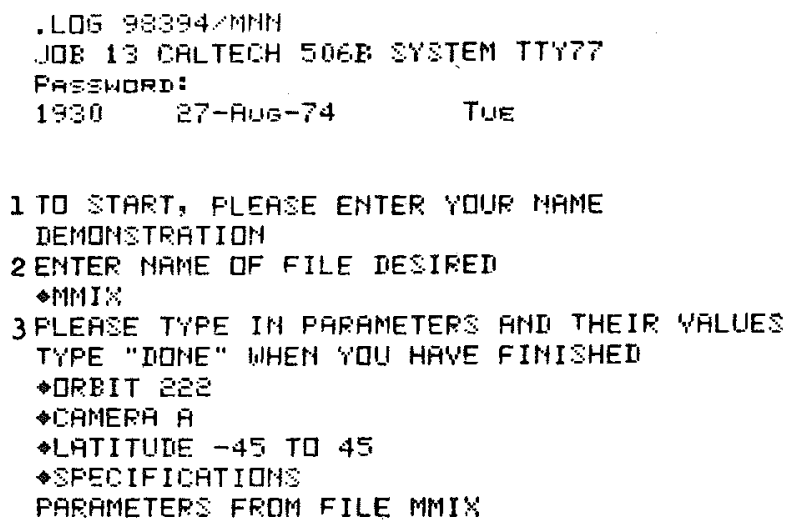


to an accounting file. The user now enters the picture set selection phase. In the current system, only two files (picture sets) are stored and the user is simply presented with a frame (2) listing the file names and giving a short description of what is contained in each. The user types the desired file name (MMIX-Mariner 9 Mars photographs) and thus enters the parameter specification phase. The available selection parameters and acceptable values are now shown (3). The user specifies some param-

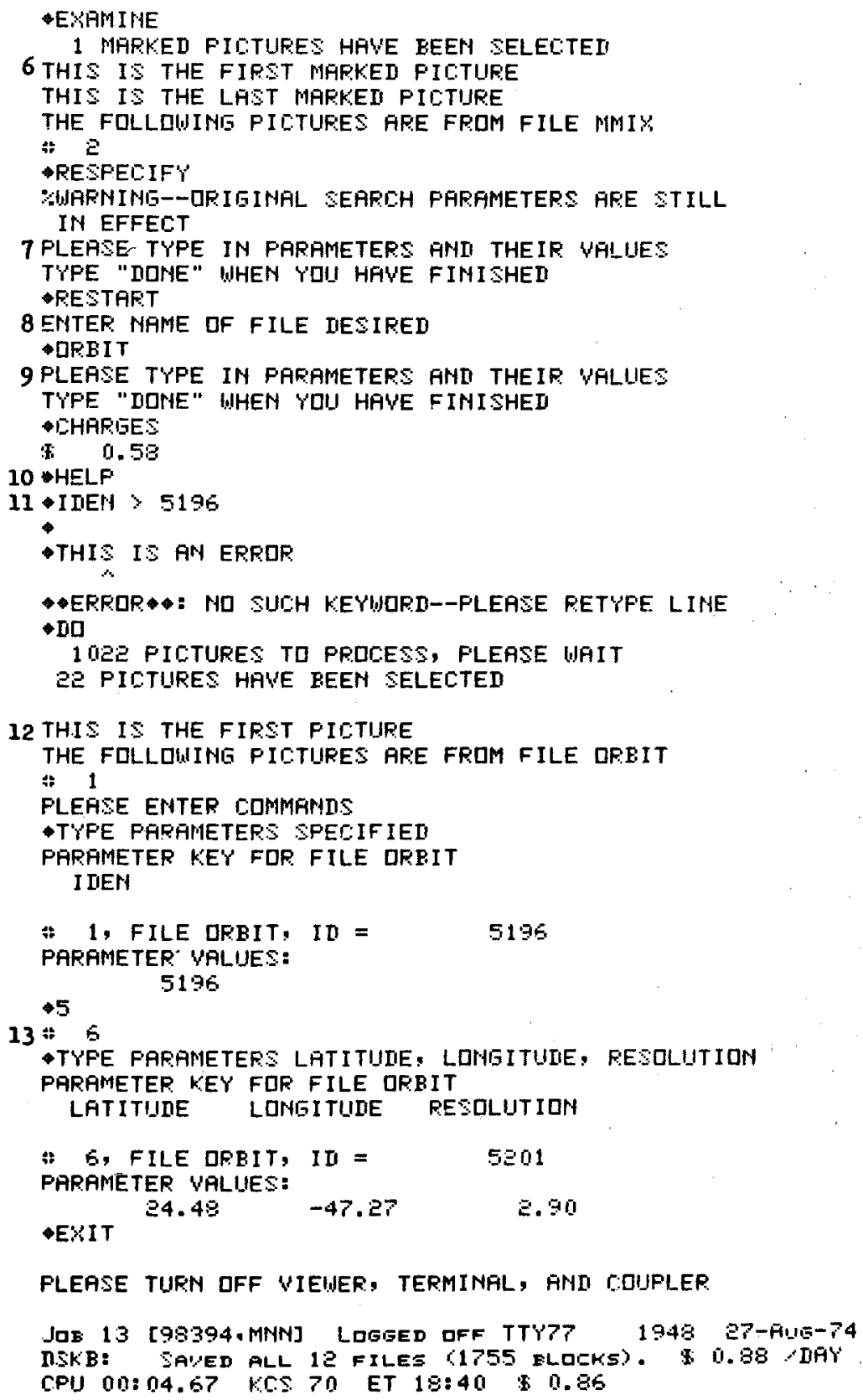


eters, then asks the program to verify what he has entered. He can change any specifications he wishes or return to the picture set selection phase. When he wishes the optimization and search phases to begin, he types DONE. The search shown only took about two seconds. The program is now in the picture display phase. The first selected picture (4) is shown. The user moves on to the next picture (5) by typing a carriage return. He "marks" the current picture, then requests that all the parameters of all the selected marked pictures be typed out. The program types a heading, the manual key code for the picture and associated pictures (the viewer can also be operated by push buttons on its front panel), and then types the parameter values. EXAMINE calls for only the marked pictures to be displayed. The user then decides to respecify selection parameters, changes his mind and decides to go to a different picture set, and then makes another search.

This example only demonstrates some of the available commands; a complete list is given in Appendixes 3 and 4.

\section{APPENDIX 2}

The Format Record

Entry for Each Parameter Field

Field

(by array name)

FORM

HEADER

ALFPAM

INFO

VTYPE

FORMAT

File Description Fields

Field.

(by COMMON name)

RECDAT

BINDAT

VERS
Includes entries for the number of picture records in the file, the number of parameters in a picture record, and the length of a picture record.

Position in a picture record of the value for each of the parameters.

Processing code, allows each parameter to be processed by a different method.

Ten-character alphanumeric description of the parameter.

Viewer control code for frame containing additional information about the parameter.

External form of the parameter, whether real or integer.

FORTRAN Field Specification for output of the parameter.

For the search optimization phase, tells for which parameters information is available for restricting the search. Includes an entry for each such parameter containing: (1) parameter number; (2) the average number of picture records per unit of the parameter value; (3) the lowest and highest value - in the fileof the parameter; (4) the position-in a picture record - of the parameter; (5) a code for whether the picture file or a control list is to be used to control the comparison search; and (6) the file name of the control list if it is used.

If, for each picture in the file, there are others which differ by only one characteristic, they are called versions of a picture. If all versions are placed in a certain order, and a record is . kept 
of this order, then only one picture record is necessary for all versions. This field has an entry for each version. The entries contain: (1) a keyword by which the version is referred to; (2) a pointer to the position in a picture table entry of the viewer control code of a "base" picture; and (3) the horizontal and vertical displacement on a microfiche card of the version relative to the base picture.

AUXIL Contains an entry for each type of data picture. An entry consists of the keyword description of the type of picture and of a pointer to the position of the viewer control code for the picture in a picture table entry.

COMMD For user commands which are to be valid only for this file. An entry contains a keyword and a branch code for each such command.

\section{APPENDIX 3}

\section{Immediate Commands}

These commands may be used at any time an asterisk has been given by the program.

Keyword Command

EXIT

HELP

SPECIFICATIONS

$\mathrm{Hn}, \mathrm{Vn}$

NOTE

HINT

INFORMATION $\mathbf{n}$

COMMENTS

VERSION Keyword

CHARGES

STORE $n$

SHOW n
Function

Used to stop the program.

Displays a table of contents of the instruction frames and how to display any of them.

Types out the parameters and values specified.

The pictures on a microfiche card are arranged in rows and columns. Any picture on the currently displayed card may be viewed by moving horizontally and vertically relative to the present card position. Hn moves ' $\mathrm{n}$ ' positions horizontally, Vn moves ' $\mathrm{n}$ ' vertically. $\mathrm{n}$ may be negative. If both $\mathrm{H}$ and $\mathrm{V}$ are used, they are separated with a comma. For example: V-3, H5 means move up 3 , move 5 to the right. (Positive and negative numbers indicate opposite directions.)

The rest of the input line is ignored. Used to insert information to be remembered into the typewritten record.

Allows the user to enter comments into a disc file which can later be read by the personnel responsible for system maintenance.

Provides further information for parameter $\mathbf{n}$ (such as maps, explanations, etc.)

Used to obtain a list of the available picture content descriptors. Equivalent to the INFORMATION command with the comments parameter.

Used to select different versions of the same picture. "Keyword" identifies the version. Another instruction page explains which version is initially displayed as well as the keywords for other available versions.

Types the charges accumulated since starting.

$1 \leq n \leq 15$. The currently displayed picture is saved at storage location $n$ by STORE $n$ and can later be recalled by SHOW $n$. 


\section{APPENDIX 4}

Picture Display and Information Access Commands

Display commands are used after the pictures have been selected. The selected pictures are stored in a list. The first picture displayed is the first picture in the list. Depressing the carriage return key always selects the next picture. To display any picture in the list, the number of that picture relative to the currently displayed picture is typed. For example, to view the third picture if the current picture is the tenth, -7 is typed. Typing 0 again displays the current picture if an immediate command or a data frame command have been used to display another frame. Items in parentheses are options.

Further Commands

Display

FIRST

LAST

NUMBER

MARK (ALL)

CLEAR (ALL)

EXAMINE

\section{REMARK}

KEEP

Information Access

Data Frame Display

TYPE

TYPE ID (ALL)

(MARKED)

TYPE MANUAL (ALL)

(MARKED)

$\begin{array}{lll}\text { TYPE PARAMETERS (FILE File Name) } & \text { (ALL) } & (\text { SPECIFIED) } \\ \text { (MARKED) } & \left(\mathrm{n}_{1}, \mathrm{n}_{2}, \ldots \mathrm{n}_{\mathbf{n}}\right)\end{array}$
Displays the first picture in the list; a large negative number is equivalent.

Displays the last picture in the list; a large number is equivalent. Types the position of the current picture in the list. This is given automatically whenever a picture is displayed.

Marks the current picture (or all pictures) for further processing. Clears the mark, if present.

Display only pictures which have been marked; the first is the first marked.

The converse of EXAMINE, display all pictures in the list (relative to the current picture). This is the original state of the system.

Keep marked pictures on the selected picture list through the next search. The marked pictures are moved to the beginning of the list with the marks cleared. The unmarked pictures are unavailable.

To display a data frame to the currently displayed picture, a keyword command is typed. The commands are one-word descriptions of the data frames. Different commands may apply to each picture set.

To type out information on the I/O terminal. The TYPE command has various forms indicating what is to be typed out and for which pictures. The ID number, the code for selecting a picture manually, and the parameter values of the picture(s) can be requested. This request can optionally apply to the currently displayed picture, the marked pictures, or all the selected pictures. For a request to type parameter values it is possible to specify parameters. Words in parentheses are optional. The forms of the TYPE command are: 
If not used, file name is assumed to refer to the file last searched. If the parameters are not enumerated, those specified for the picture selection are typed out. The parameters to be typed out can be enumerated or the specification parameters called for. If neither of these is done, the values of all parameters are typed out. Parameters typed out are identified by column headings.

Phase Transfer Commands

Function

RESPECIFY

Allows respecification of selection parameters-only those parameters which are reentered are changed; previously specified parameters retain their values.

SEARCH

Similar to RESPECIFY, except only those pictures in the present list are candidates for selection. This is more efficient than again searching through all the pictures.

CONTINUE

RESTART If the search was terminated before all pictures had been processed, the search is continued from where it had been suspended.

To view another set of pictures (all specified parameter values are deleted).

\section{APPENDIX 5}

Mariner 9 Picture Records

Field Number

Field

Fixed-Length Portion

1 Fiche Code

2 Data Code

3 File Name

4 ID Number (DAS)

$5 \quad$ Unit \#

$6 \quad$ Picture Number

$7 \quad$ Footprint Code

8 Unused

9

Variable Portion

10 Orbit

11 Latitude

12 Longitude

13 Solar Lighting Angle

14 Phase Angle

15 Viewing Angle

16 Slant Range

17 Camera

18 Resolution

19 Local Time

20 Filter

21 Exposure Time

22 Role and File of Filter Version on Roll Film

23-28 Comments (Content Descriptors) 